\title{
Automatic 3D to 2D Registration for the Photorealistic Rendering of Urban Scenes *
}

\author{
Lingyun Liu and Ioannis Stamos \\ Department of Computer Science, Graduate Center and Hunter College \\ City University of New York, New York, NY 10021 \\ lliu1@gc.cuny.edu, istamos@hunter.cuny.edu \\ Computer Vision and Pattern Recognition 2005
}

\begin{abstract}
This paper presents a novel and efficient algorithm for the $3 D$ range to $2 D$ image registration problem in urban scene settings. Our input is a set of unregistered $3 D$ range scans and a set of unregistered and uncalibrated $2 D$ images of the scene. The $3 D$ range scans and $2 D$ images capture real scenes in extremely high detail. A new automated algorithm calibrates each $2 D$ image and computes an optimized transformation between the $2 D$ images and $3 D$ range scans. This transformation is based on a match of $3 D$ with $2 D$ features that maximizes an overlap criterion. Our algorithm attacks the hard $3 D$ range to $2 D$ image registration problem in a systematic, efficient, and automatic way. Images captured by a high-resolution $2 D$ camera, that moves and adjusts freely, are mapped on a centimeter-accurate $3 D$ model of the scene providing photorealistic renderings of high quality. We present results from experiments in three different urban settings.
\end{abstract}

\section{Introduction}

This paper deals with the problem of automatic pose estimation and calibration of a 2D camera with respect to an acquired geometric 3D model of an urban scene. The pose estimation is part of a larger system which constructs highresolution photorealistic 3D models from unregistered 3D range scans and uncalibrated 2D color images. Our goal is to enhance the geometric model with photographic observations taken from a freely moving $2 \mathrm{D}$ camera by automatically recovering the camera's position and orientation with respect to the model of the scene and by automatically calibrating the camera sensor. We are attacking the stated problem under the following assumption: the 3D scene contains 3D lines defining two major orthogonal directions, i.e. one major vertical direction and at least one major horizontal

${ }^{*}$ Supported in part by NSF CAREER IIS-01-21239, NSF MRI/RUI EIA-0215962. We thank Prof. George Wolberg and his group for the acquisition of building 3 dataset. direction. This is a valid assumption that represents the large majority of scenes in urban settings.

Most systems recreating photorealistic models of the environment by a combination of range and image sensing $[2,7,19,21,26]$ solve the $3 \mathrm{D}$ range to $2 \mathrm{D}$ image registration problem by fixing the relative position and orientation of the camera with respect to the range sensor (the two sensors are rigidly attached on the same platform). The fixed-relative position approach provides a solution that has the following major limitations: A) The 3D-range and 2Dimage captures occur at the same point in time and from the same location in space. That leads to a lack of 2D sensing flexibility, since the limitations of 3D-range sensor positioning (standoff distance, maximum distance) will cause constraints on the 2D camera placement. Also, the 2D images may need to be recaptured due to poor lighting conditions at the time of the 3D-range capture. B) The static arrangement of $3 \mathrm{D}$ and $2 \mathrm{D}$ sensors also means that the 2D camera can not be dynamically adjusted (by changing its focal length and position) to the requirements of each particular scene. C) The fixed approach can not handle the case of mapping historical photographs on the models or of mapping 2D images captured at a different instant in time (under different lighting conditions), something that our method is able to accomplish. In summary, by fixing the relative position between the 3D-range and 2D-sensors, we sacrifice the flexibility of 2D-image capturing. We believe that methods similar to the one provided in this paper are essential for the accurate photorealistic capture of urban scenes.

This work is a continuation of our original contributions on the subject of $3 \mathrm{D}$ range to $2 \mathrm{D}$ image registration $[22,23]$. In previous work, we provided a solution for automatically matching $3 \mathrm{D}$ and $2 \mathrm{D}$ features from the range and image datasets. The approach involved the utilization of parallelism and orthogonality constraints that naturally exist in urban environments. This paper is based on our original framework, but a number of novel avenues are now explored. Here are our new contributions: $i$ ) Extraction of a richer set of 3D features by utilizing data from all reg- 
istered 3D range images at once. ii) Utilization of new type of higher-order clusters of 3D and 2D features. iii) Development of a new method for optimizing the internal camera parameters. iv) Development of a new algorithm for matching 3D with 2D features. Here, we would like to point out that our new algorithm is not a probabilistic RANSAC approach. The whole search space is efficiently and systematically explored. $v$ ) Development of user-interface for minimal user interaction.

There are many approaches for the solution of pose estimation problem from both point correspondences $[17,20]$ and line correspondences $[5,10]$, when a set of matched 3D and 2D points or lines are known, respectively. In the early work of [6], the probabilistic RANSAC method for automatically computing matching $3 \mathrm{D}$ and $2 \mathrm{D}$ points was introduced. This approach works well only when the percentage of outliers (i.e. incorrectly matched pairs) is small. Works in automated matching of 3D with 2D features in context of object recognition and localization includes $[3,9,11,13,14,18,25]$. Recently, a scale-invariant approach in the context of 2D image registration has been presented in [16]. Teller [1] attacks the 2D image registration problem in urban environment settings as well. In [27], Zhao uses SFM and stereo algorithms to map a continuous video from an aerial source on a 3D urban model. Ikeuchi [12] presents an automated 3D range to $2 \mathrm{D}$ image registration method that relies on the reflectance range image. In our work, we attack the $3 \mathrm{D}$ range to $2 \mathrm{D}$ image registration problem in an efficient and systematic way (i.e. we do not rely exclusively on 2D images). In order to achieve this, we have implemented novel feature extraction and 3D range to 2D image matching techniques.

\section{3D Feature Extraction}

The first step is to acquire $n$ range scans to adequately cover the 3D scene. The laser-range scanner is Cyrax 2500 [15], an active sensor that emits eye-safe laser beams into the scene. It is capable of gathering one million 3D points at a maximum distance of 100 meters. A range scan of an urban scene is shown in (Fig. 1(a)). Each point is associated with four values $(x, y, z, l)^{T}$, where $(x, y, z)^{T}$ is its Cartesian coordinates in the scanner's local coordinate system, and $l$ is the laser intensity of the returned laser-beam. The intensity depends on: the material properties of the physical 3D surface, the distance of the point from the range sensor, and the orientation of the laser beam with respect to the local surface normal at the measured 3D point.

Each range scan is processed via an automated segmentation algorithm [23]. A set of major 3D planes and a set of geometric 3D lines $G_{i}$ are extracted from each scan $i=1, \ldots, n$. The geometric 3D lines are computed at the intersections between segmented planar regions and at the borders of the segmented planar regions [23]. The range scans are registered in the same coordinate system via the automated range-range feature-based registration method which is described in $[4,24]$. As a result, all range scans are registered with respect to one selected pivot scan, in the scene's coordinate system, namely $S_{3 D}$. In addition to the geometric lines $G_{i}$, a set of reflectance 3D lines $L_{i}$ are extracted from each 3D range scan. They are produced by discontinuities of the laser intensity (Fig. 1(a)). We extract 2D lines from the reflectance image using standard image processing techniques (Canny edge detector followed by orthogonal regression). The end points of each reflectance

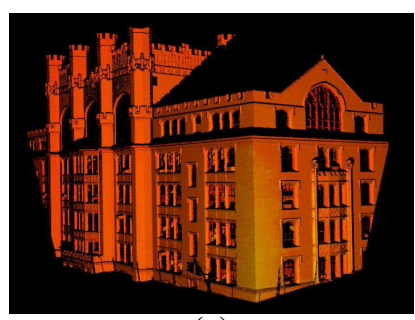

(a)

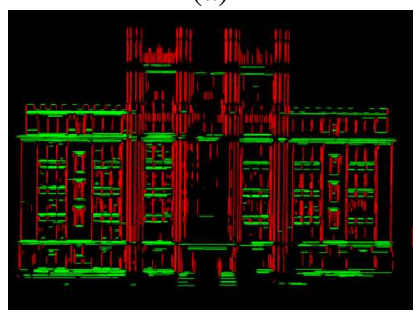

(c)

(e)

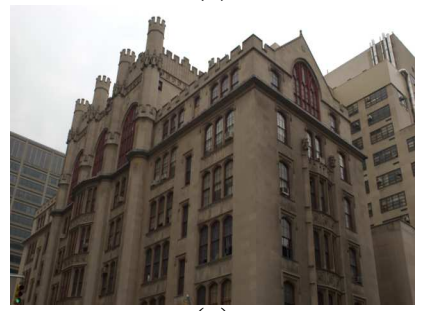

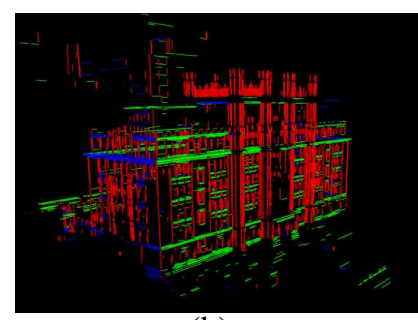

(b)

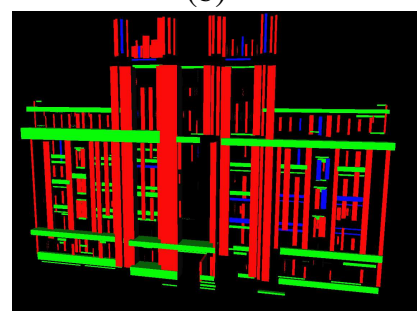

(d)

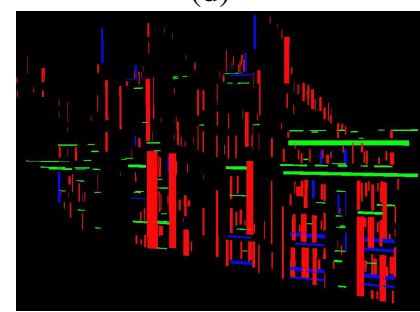

(f)
Figure 1. (a) A range-scan of building 1 at a resolution of one-million 3D points (Cyrax 2500 laser scanner).The pseudo-color of each point corresponds to the returned laser intensity. (b) Clustered line sets for building 1 (12 range scans). Three major directions are identified with different colors: red for vertical, green and blue for two horizontal directions. (c) Lines on a 3D face of building 1 (vertical and one horizontal directions are used). (d) Vertical and horizontal 3D parallelepipeds for the 3D face of building 1 in (c). (e) A 2D image of building 1. (f) Vertical and horizontal rectangles from the rectified lines which are extracted from the 2D image in (e). Two vanishing points are used. In images (d) and (f), the matching 3D and 2D features, as extracted by our automated algorithm, are highlighted in blue.

2D line $\left(p_{1}, p_{2}\right)$ correspond to two 3D points $\left(x_{1}, y_{1}, z_{1}\right)$ and $\left(x_{2}, y_{2}, z_{2}\right)$ which define a geometric line in 3D space. We call this line a reflectance 3D line because it is computed based on information gathered from the reflectance 
image alone.

The combination of all 3D geometric and reflectance lines provides a very rich representation of the acquired 3D scene. We use $\mathcal{L}^{3 D}$ to represent those lines. Therefore: $\mathcal{L}^{3 D}=\bigcup_{i} L_{i}^{\prime} \cup G_{i}^{\prime}$, where $L_{i}^{\prime}$ and $G_{i}^{\prime}$ are the computed reflectance lines $L_{i}$ and geometric lines $G_{i}$ of each scan after their transformations into the scene coordinate system $S_{3 D^{1}}$.

The next step is to cluster the line set $\mathcal{L}^{3 D}$. Each line in a cluster has the similar orientation as every other line in the same cluster. One large cluster of vertical 3D lines and a number of clusters of horizontal 3D lines are expected to be obtained. We call the cluster of vertical lines $\mathcal{L}^{3 D}{ }_{v}$, and the one (or more) clusters of horizontal lines $\mathcal{L}^{3 D}{ }_{h_{1}}, \mathcal{L}^{3 D}{ }_{h_{2}}, \ldots, \mathcal{L}^{3 D}{ }_{h_{m}}$. Figs. 1 (b) shows the clustered sets of 3D geometric and reflectance lines from one of our experiments.

In all the following algorithms, 3D and 2D lines or features are transformed to the coordinate system $S_{b a s e}$. $S_{\text {base }}$ serves as a common intermediate coordinate system of reference, where the horizontal features are parallel to the $\mathrm{x}$ axis and the vertical features parallel to the y-axis. That property makes the implementations of feature matching and translation computation very efficient (see section 4). $S_{\text {base }}$ is defined by the three orthogonal axes $\hat{\mathbf{x}}=[1,0,0]^{T}$, $\hat{\mathbf{y}}=[0,1,0]^{T}, \hat{\mathbf{z}}=[0,0,1]^{T}$, and the origin which is at $[0,0,0]^{T}$.

Let us consider one pair of vertical and horizontal clusters $\left(\mathcal{L}^{3 D}{ }_{v}, \mathcal{L}^{3 D}{ }_{h_{i}}\right)^{2}$. The 3D lines of this pair are aligned to the axes of the coordinate system $S_{\text {base }}$ through a rotation $R_{3 D}(i): R_{3 D}(i)=[\hat{\mathbf{x}} \hat{\mathbf{y}} \hat{\mathbf{z}}]^{T} \cdot\left[\mathbf{x}_{\mathbf{n}} \mathbf{y}_{\mathbf{n}} \mathbf{z}_{\mathbf{n}}\right]^{-T}$, where $\mathbf{y}_{\mathbf{n}}=$ direction of $\mathcal{L}^{3 D}{ }_{v}, \mathbf{z}_{\mathbf{n}}=\mathbf{y}_{\mathbf{n}} \times$ direction of $\mathcal{L}^{3 D}{ }_{h_{i}}$, and $\mathbf{x}_{\mathbf{n}}=\mathbf{y}_{\mathbf{n}} \times \mathbf{z}_{\mathbf{n}}$. The transformed 3D lines are further clustered into major 3D planes. We call each one of these clusters a 3D face. Each 3D face is thus defined by its base-plane and all lines that lie on it. The lines can be either vertical or horizontal but their distances from the base-plane should be smaller than a user-defined threshold $\mathcal{B}_{\text {th }}$.

\subsection{Rectangular Parallelepiped Extraction}

Our goal is to obtain 3D features from the 3D line sets that are matchable with 2D features from the 2D color images. Matching individual 3D lines with individual 2D lines is impractical due to the large size of the generated search space. Another problem is that some 3D lines are not present in the $2 \mathrm{D}$ image and vice versa (eg. 2D lines that are generated by shading discontinuities are not present in the 3D model of the scene). Therefore, we use higher level features, i.e. vertical or horizontal 3D rectangular

\footnotetext{
${ }^{1}$ Note that we know the rotational and translational transformation between range scans from our range-range registration module.

${ }^{2}$ This pair can be interactively selected by the user via a simple colorbased user interface.
}

parallelepipeds that can be matched with $2 \mathrm{D}$ rectangles obtained from the 2D images.

The rich set of geometric and reflectance lines in a 3D face (see section 2) are grouped into sets of lines which define $3 \mathrm{D}$ rectangular parallelepipeds ${ }^{3}$ in space. The set of extracted parallelepipeds for a 3D face is called $R P$. Each parallelepiped $r p \in R P$ contains clusters of nearby $3 \mathrm{D}$ lines. There are two types of clusters: vertical (containing lines parallel to the vertical direction) and horizontal (containing lines parallel to the horizontal direction). $r p$ is thus defined by three attributes: 1) type: vertical or horizontal; 2) tl : top left vertex of $r p$; 3) br : bottom right vertex of $r p$. The computation of the set $R P$ is done as follows. Initially, every line on a 3D face becomes a trivial parallelepiped $r p$. This trivial $r p$ is a rectangle with a fixed initial width. When projections of two rp on the 3D face base-plane overlap, these two rp are merged into a bigger $r p$ which includes all lines in them. This merging process will continue until there is no overlap between any of the remaining rp. See Fig.1(d) for results.

\section{2D Feature Extraction, Internal Camera Calibration, \& Rotation Computation}

The internal parameters (focal length and principal point) of the camera sensor can be calculated from a 2D image, if the image contains at least two vanishing points (i.e. the $3 \mathrm{D}$ scene which the camera is viewing has at least two major scene directions). We use our previously developed robust methods to generate and cluster $2 \mathrm{D}$ lines from a $2 \mathrm{D}$ image [22]. The result is a set of major vanishing points $\mathbf{V}_{1}, \mathbf{V}_{2}, \cdots, \mathbf{V}_{n}$. Using the methods described in [22] we can compute the center of projection $\mathbf{C}=\left[C_{x}, C_{y}, C_{z}\right]^{T}$ (effective focal length and principal point expressed in pixels) by utilizing three orthogonal vanishing points. In the case that the scene contains only two vanishing points, we calculate the center of projection as follows. The two vanishing points $\mathbf{V}_{1}$ and $\mathbf{V}_{2}$ are expressed in the camera coordinate system as: $\mathbf{V}_{1}=\left[\left(V_{1}\right)_{x},\left(V_{1}\right)_{y}, 0\right]^{T}, \mathbf{V}_{2}=$ $\left[\left(V_{2}\right)_{x},\left(V_{2}\right)_{y}, 0\right]^{T}$. The angle between the directions that created the vanishing points is given by the clusters of $3 \mathrm{D}$ lines (in most scenarios, it's 90 degrees). If this angle is $\theta$ radians, then $\cos (\theta)=n\left(\mathbf{C}-\mathbf{V}_{1}\right) \cdot n\left(\mathbf{C}-\mathbf{V}_{2}\right)$, where $n(\mathbf{v})$ is the unit vector parallel to vector $\mathbf{v}$. The effective focal length $C_{z}$ can be computed from the equation by assuming an approximate principal point $\left(C_{x}, C_{y}\right)$ at the center of the image. This approximation is further refined at the end of this section.

The matching of the computed vanishing points with the extracted major scene directions $\mathcal{L}^{3 D}{ }_{v}$ and $\mathcal{L}^{3 D}{ }_{h_{i}}$ can be

\footnotetext{
${ }^{3}$ Note that we are extracting parallelepipeds instead of rectangles (a rectangle can be viewed as a parallelepiped with zero width). We can not expect all 3D lines to lie exactly on top of the 3D face base-plane; some of the lines are produced by architectural details on each facade, or by window frames. Therefore, the extracted linear features of each 3D face may lie as far as $\mathcal{B}_{t h}$ meters away from the major scene facade.
} 
automatically achieved by using a-priori assumptions about the position of the camera with respect to the $3 \mathrm{D}$ scene, or by using information about the relative size of matched clusters. Alternatively, the user can easily pick the correct matches through a color-based user-interface. The correspondence between vanishing points $\mathbf{V}_{v}, \mathbf{V}_{h_{k}}$ and $3 \mathrm{D}$ directions $\mathcal{L}^{3 D}{ }_{v}, \mathcal{L}^{3 D}{ }_{h_{i}}$, provides a solution to the rotation $R_{2 D}$ that brings the 2D features in the coordinate system $S_{\text {base }}$ (see Sec.2): $R_{2 D}=[\hat{\mathbf{x}} \hat{\mathbf{y}} \hat{\mathbf{z}}]^{T} \cdot\left[n\left(\mathbf{v}_{1} \times \mathbf{v}_{2}\right) \mathbf{v}_{1} \mathbf{v}_{2}\right]^{-T}$, where $\mathbf{v}_{1}=n\left(\mathbf{V}_{v}-\mathbf{C}\right)$ and $\mathbf{v}_{2}=n\left(\mathbf{v}_{1} \times\left(\mathbf{V}_{h_{k}}-\mathbf{C}\right)\right)$. Then all $2 \mathrm{D}$ lines can be rectified by applying the rotation $R_{2 D}$ about $\mathbf{C}$ and then by projecting to the image plane.

The center of projection $\mathbf{C}$ can be further refined as follows. By construction, the rectified 2D lines from $\mathcal{L}^{2 D} v$ and $\mathcal{L}^{2 D}{ }_{h_{i}}$ should be parallel to the $y-$ axis and $x-$ axis of $S_{b a s e}$, respectively (Fig. 1(f)). But if the estimation of $\mathbf{C}$ is not accurate, the angle between the actual direction of a rectified line and its expected direction will not be zero. This per-line computed angle is considered to be an error due to an inaccurate $\mathbf{C}$ computation. The sum of the errors of all lines,called $\mathcal{A}$, is used as a criterion to compute a more accurate center of projection as follows. We consider all possible centers of projections in a small spatial neighborhood around the initially computed center C. Searching for the best center of projection $\mathbf{C}_{b e s t}$ is done sequentially in a spiral manner in the neighborhood of the initial C. $\mathbf{C}_{b e s t}$ is the first center of projection that produces an angle error $\mathcal{A}$ less than a user-defined threshold $\mathcal{A}_{t h}$ (normally between 2 to 5 degrees). Then we have the camera calibration matrix $\mathcal{K}$ as ([8]):

$$
\mathcal{K}=\left|\begin{array}{ccc}
-\left(C_{b e s t}\right)_{z} & 0 & \left(C_{b e s t}\right)_{x} \\
0 & -\left(C_{b e s t}\right)_{z} & \left(C_{\text {best }}\right)_{y} \\
0 & 0 & 1
\end{array}\right|
$$

The same method is applied to extract horizontal and vertical rectangular $2 \mathrm{D}$ features $R C$ as the one used to extract 3D features $R P$ in Sec.2.1, except that the extracted $2 \mathrm{D}$ features has a zero depth, i.e. they are rectangles instead of parallelepipeds.

\section{Translation Computation}

In this section we present the algorithm that automatically computes the translation between the scene $S_{3 D}$ and the camera $S_{\text {camera }}$ coordinate systems (Fig.2). From the previous steps, we have the sets of $3 \mathrm{D}$ parallelepipeds $R P$ and $2 \mathrm{D}$ rectangles $R C$ ready to be matched in $S_{\text {base }}$. The rotation $R$ that brings $S_{3 D}$ to $S_{\text {camer } a}$ is $R=R_{2 D}{ }^{-1} \cdot R_{3 D}$. The two rotational components $R_{3 D}$ and $R_{2 D}$ were computed in sections 2 and 3 , respectively.

In order to compute the translation between the scene and camera coordinate systems, we need to identify $K$ 3D parallelepipeds $r p$ (see Fig.1(d) for an example set of 3D features) that match $K$ 2D rectangles $r c$ (see Fig.1(f) for an example set of $2 \mathrm{D}$ features). It is important to note that we can only match horizontal 3D features with horizontal 2D features, and vertical 3D features with vertical 2D features. The problem is thus reduced to a $2 \mathrm{D}$ pattern matching problem. A single matched $r p$ with a $r c$ is not able

Features Matching \& Translation Computation

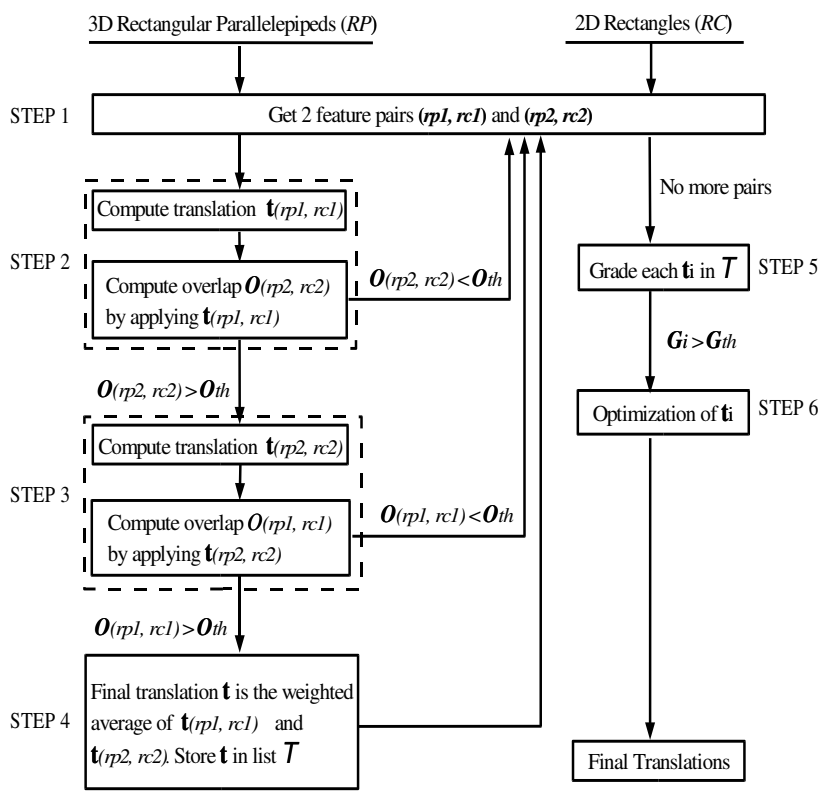

Figure 2. Translation computation algorithm outline.

to provide the correct translation as our experiments have shown. This is due to the nature of our extracted features. However, two correctly matched $r p$ with 2D $r c$ are able to provide us a very accurate translation as will be shown in the algorithm to follow. Thus our algorithm searches through all pairs of possible matches systematically. Note that we consider all $\left(\begin{array}{c}N_{h} \\ 2\end{array}\right) \times\left(\begin{array}{c}n_{h} \\ 2\end{array}\right)+\left(\begin{array}{c}N_{v} \\ 2\end{array}\right) \times\left(\begin{array}{c}n_{v} \\ 2\end{array}\right)$ possible matched pairs (where $N_{h}, N_{v}$ are the number of horizontal and vertical $r p$, respectively, and $n_{h}, n_{v}$ are the number of horizontal and vertical $r c$, respectively). This is a large search space, but it can be efficiently explored as our results show (see Sec.5).

Our algorithm consists of six steps (Fig.2). In the first four steps, a list of candidate translations are being computed from the exploration of all possible matches between pairs of 3D parallelepipeds and pairs of $2 \mathrm{D}$ rectangles. The fifth step determines the grades of each candidate translations based on the number of matching 3D and 2D feature pairs produced by this translation. The candidates with grades smaller than a threshold will be eliminated. The sixth step searches in the neighborhood of each remaining candidate translation for the one that maximizes the amount of overlap. Thus, for each candidate, a final optimized translation $\mathbf{t}_{\text {opt }}$ is computed.

For a 3D feature (parallelepiped) pair rp = $\left(r p_{1}, r p_{2}\right) \in R P \times R P$, and a $2 \mathrm{D}$ feature (rectangle) pair 
$\mathbf{r c}=\left(r c_{1}, r c_{2}\right) \in R C \times R C, \mathbf{c}$ is defined as the centroid of a $3 \mathrm{D}$ or $2 \mathrm{D}$ feature, while $\pi_{3 D}$ is the base-plane of $3 \mathrm{D}$ face where rp lie on and $\pi_{2 D}$ is the 2D image plane (note that these planes are parallel to the x-y plane of the $S_{\text {base }}$ coordinate system).

(Step 1) Consider the next pair of 3D features rp $=$ $\left(r p_{1}, r p_{2}\right)$ to match the pair of $2 \mathrm{D}$ features $\mathbf{r c}=\left(r c_{1}, r c_{2}\right)$. All possible matching pairs are considered. Many of them can be discarded as shown in the following steps.

(Step 2) We assume that the 3D feature $r p_{1}$ matches the $2 \mathrm{D}$ feature $r c_{1}$. Then the translation vector $\mathbf{t}_{\left(r p_{1}, r c_{1}\right)}$ that brings the centroids of both features into alignment should satisfy the following equation (see Fig.3): $\mathbf{c}_{r c_{1}}=$ $\mathcal{K}\left[\mathbf{I} \mid \mathbf{t}_{\left(r p_{1}, r c_{1}\right)}\right] \mathbf{c}_{r p_{1}}$, where $\mathbf{I}$ is identity matrix. $\mathbf{t}_{\left(r p_{1}, r c_{1}\right)}$ is computed as follows. First, we compute the ratio $p m=$ $\frac{l e n_{r p_{1}}}{l e n_{r c_{1}}}$, where len indicates length of the feature (horizontal or vertical length depending on feature's type). This

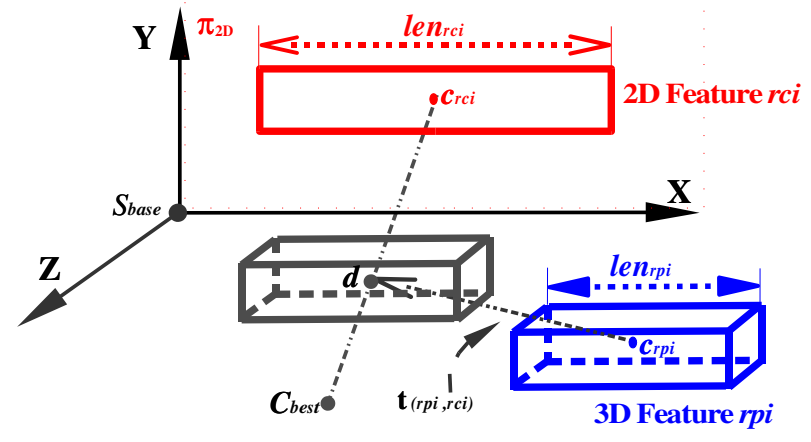

Figure 3. Translation computation.

ratio reflects the scale that should be applied to the $2 \mathrm{D}$ image feature (measured in pixels), so that it matches the 3D feature (measured in meters). Then, considering the line segment from the centroid of the $2 \mathrm{D}$ feature $\mathbf{c}_{r c_{1}}$ to the center of projection $\mathbf{C}_{b e s t}$, we find the point $\mathbf{d}$ so that $\frac{\left\|\mathbf{C}_{\text {best }}-\mathbf{d}\right\|}{\left\|\mathbf{C}_{\text {best }}-\mathbf{c t}_{r c_{1}}\right\|}=p m$, where $\|\mathbf{v}\|$ denotes the norm of vector $\mathbf{v}$. The translation vector $\mathbf{t}_{\left(r p_{1}, r c_{1}\right)}$ can now computed as follows (Fig.3): $\mathbf{t}_{\left(r p_{1}, r c_{1}\right)}=\mathbf{d}-\mathbf{c}_{r c_{1}}$. This translation vector translates the $3 \mathrm{D}$ feature $r p_{1}$ (note that $r p_{1}$ is already expressed in the common coordinate system $S_{\text {base }}$ ) into the unique position that makes its projection to the image plane $\pi_{2 D}$ have the following properties: A) The length of the projection of $r p_{1}$ is exactly the same as the length of $r c_{1}$, and B) The centroid of $r p_{1}$ is projected exactly on the centroid of $r c_{1}$. The estimation of this translation vector can be performed very efficiently in the coordinate system $S_{\text {base. }}$. This is one of the factors that attributes to the efficiency of our algorithm.

The just obtained translation brings the two features $r p_{1}$ and $r c_{1}$ into alignment (the center of $r p_{1}$ is projected on the center of $r c_{1}$ ). A correct translation will also bring $r p_{2}$ and $r c_{2}$ into alignment if these 2 features are corresponding to each other. By applying the translation $\mathbf{t}_{\left(r p_{1}, r c_{1}\right)}$ to $r p_{2}$, and projecting it onto $\pi_{2 D}$, we produce a $2 \mathrm{D}$ feature called $r p_{2}{ }^{p}$ (this is a 2D rectangle): $r p_{2}{ }^{p}=\mathcal{F}\left(r p_{2}, \mathbf{t}_{\left(r p_{1}, r c_{1}\right)}\right)$, where $\mathcal{F}$ is the function that projects $3 \mathrm{D}$ features onto $\pi_{2 D}$. This is achieved by projecting two translated diagonal vertices (top left and bottom right) of the $3 \mathrm{D}$ feature onto $\pi_{2 D}$ and then generating 2D rectangle based on the two projected vertices. The percentage of overlap among the features $r p_{2}{ }^{p}$ and $r c_{2}$ is denoted as $\mathcal{O}_{\left(r p_{2}{ }^{p}, r c_{2}\right)}$. If this overlap is larger than a user-defined threshold $\mathcal{O}_{t h}$ (normally $80 \%$ ), we proceed to the next step; otherwise we go back to step 1 , and have the next pair of features to be matched.

(Step 3) Step 2 is repeated for the computation $\mathbf{t}_{\left(r p_{2}, r c_{2}\right)}$ (we now assume that $r p_{2}$ matches $r c_{2}$ ). If the overlap $\mathcal{O}_{\left(r p_{1}{ }^{p}, r c_{1}\right)}$ is larger than $\mathcal{O}_{t h}$ as well, the two pairs $(\mathbf{r p}, \mathbf{r c}) \in R P^{2} \times R C^{2}$ are considered as matching candidates (otherwise the next pair of matches is considered at step 1).

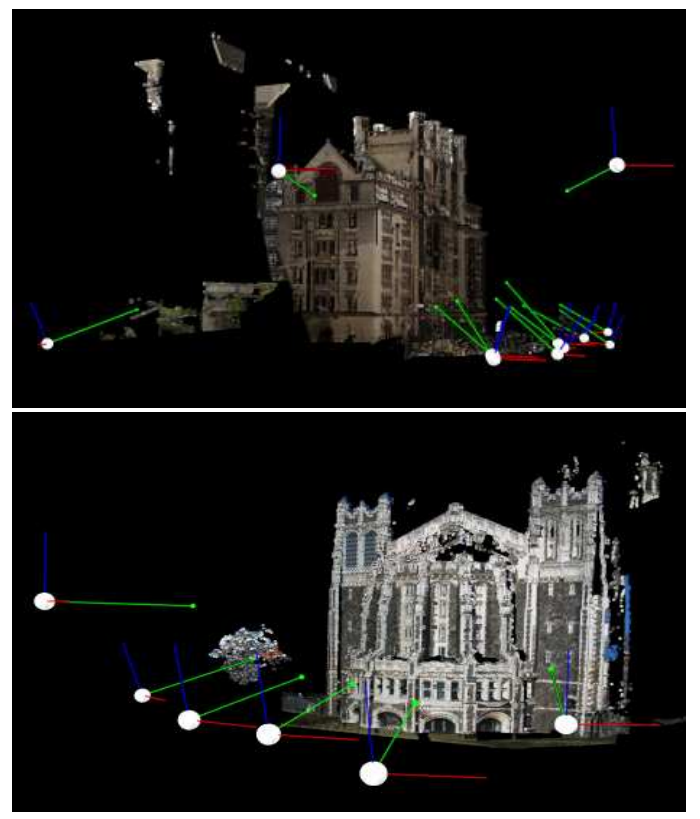

Figure 4. Camera configurations with respect to texturemapped 3D models of buildings 1 (top) and 3 (bottom). The white dots are the locations of the automatically recovered cameras, and the green axes are the sensing directions of the cameras. For building 1, nine ground-level cameras are recovered. Two images are shot from balconies of nearby buildings. For buildings 2 and 3, six images are recovered respectively.

(Step 4) The final translation $\mathbf{t}_{(\mathbf{r p}, \mathbf{r c})}$ is computed by a weighted average of the component translations: $\mathbf{t}_{(\mathbf{r p}, \mathbf{r c})}=$ $w_{1} \cdot \mathbf{t}_{\left(r p_{1}, r c_{1}\right)}+w_{2} \cdot \mathbf{t}_{\left(r p_{2}, r p_{2}\right)}$, where the weight is the overlap area ratio (i.e. the bigger the relative overlap, the bigger the weight): $w_{1}=\frac{\mathcal{O}_{\left(r p_{2} p^{p} r c_{2}\right)}}{\mathcal{O}_{\left(r p_{1}{ }^{p}, r c_{1}\right)}+\mathcal{O}_{\left(r p_{2}{ }^{p}, r c_{2}\right)}}, w_{2}=1-$ $w_{1}$.

(Step 5) By repeating steps 1 through 4 on all possible pairs of 3D and 2D feature pairs $(\mathbf{r p}, \mathbf{r c}) \in R P^{2} \times R C^{2}$, the translations of all matching candidates are computed 
and stored in a set $\mathcal{T}$. Each translation $\mathbf{t}_{i}$ in $\mathcal{T}$ is applied to all $r p \in R P$. The translated $r p$ are then projected on $\pi_{2 D}$, producing rectangles $r p^{p}$. Every pair $\left(r p^{p}, r c\right)$ with overlap larger than $\mathcal{O}_{t h}$ is stored in the set $\mathcal{M}_{i}$. The total number of the pairs in $\mathcal{M}_{i}$ is defined as the grade $\mathcal{G}_{i}$ of the translation $\mathbf{t}_{i}$. $\mathbf{t}_{i}$ in $\mathcal{T}$ are then sorted based on $\mathcal{G}_{i}$, and the translations with grade less than a user-defined threshold $\mathcal{G}_{t h}$ are deleted from the list ${ }^{4}$. This increases the efficiency, without sacrificing the accuracy of the algorithm.

(Step 6) Each translation $\mathbf{t}_{i}$ in the list $\mathcal{T}$ can be optimized even further. Our goal is to maximize the overlap between matching pairs produced by each translation $\mathbf{t}_{i}$ (the set of matching features for each translation has been stored in $\mathcal{M}_{i}$ - see previous step). We consider a neighborhood $\mathcal{N}_{i}$ of all possible translations around $\mathbf{t}_{i}$. For each one of the translations in this neighborhood, the overlap between the matching features in $\mathcal{M}_{i}$ is calculated. The translation in $\mathcal{N}_{i}$ that produces the maximum overlap is chosen as our final optimum result for $\mathbf{t}_{i}$ : $\mathbf{t}_{\text {opt }}(i)=$ $\max _{\mathbf{t} \in \mathcal{N}_{i}}\left(\sum_{(r p, r c) \in \mathcal{M}_{i}} \mathcal{O}_{(\mathcal{F}(r p, \mathbf{t}), r c)}\right)$.

As a result, the user is presented with a sorted list of $\mathbf{t}_{\text {opt }}(i)$. In most cases, the translation with the highest grade (i.e. largest number of matching features) is the one that produces the best result. Otherwise, user can select the $2 \mathrm{nd}$, 3rd or other listed translation. This selection is extremely simple, since user can instantly assess the computed translation by visually inspecting the computed texture mapped result.

Finally, a point $\mathbf{x}_{3 D}$ can be transformed from the $3 \mathrm{D}$ scene coordinate system to a point $\mathbf{x}_{\text {cam }}$ in the $2 \mathrm{D}$ camera coordinate system via:

$$
\mathbf{x}_{\text {cam }}=\mathcal{K}\left[R \mid\left(R_{2 D}{ }^{-1} \mathbf{t}_{o p t}(i)\right)\right] \mathbf{x}_{3 D}
$$

\section{Results and Conclusions}

We performed experiments in three urban settings (buildings 1, 2 and 3). Buildings 1 and 2 are regular urban structures with many windows and large planes (a photograph of building 1 is shown in Fig.1(a)), while building 3 has a more complicated structure (as shown in Fig. 5 bottom two images). A number of 3D range scans and 2D images were acquired for each building. After the range scans are registered on the same coordinate system, the $2 \mathrm{D}$ images are automatically calibrated and registered on the 3D model of each acquired structure. Fig. 4 presents overviews of our texture-mapping results and automatically recovered camera configurations. We have not performed any intelligent blending of overlapping 2D images, something that will be part of our future work. Finally, detailed textured maps are shown in Figs.5. Two of the automated transformations computed from building 3 required a very small correction

\footnotetext{
${ }^{4}$ The grade threshold $\mathcal{G}_{t h}$ is computed as $\mathcal{G}_{t h}=\mathcal{G}_{\min }+\alpha \cdot\left(\mathcal{G}_{\max }-\right.$ $\mathcal{G}_{\text {min }}$ ), where $\mathcal{G}_{\max }$ and $\mathcal{G}_{\text {min }}$ are the maximum and minimum grades, $0.6 \leq \alpha \leq 1.0$.
}

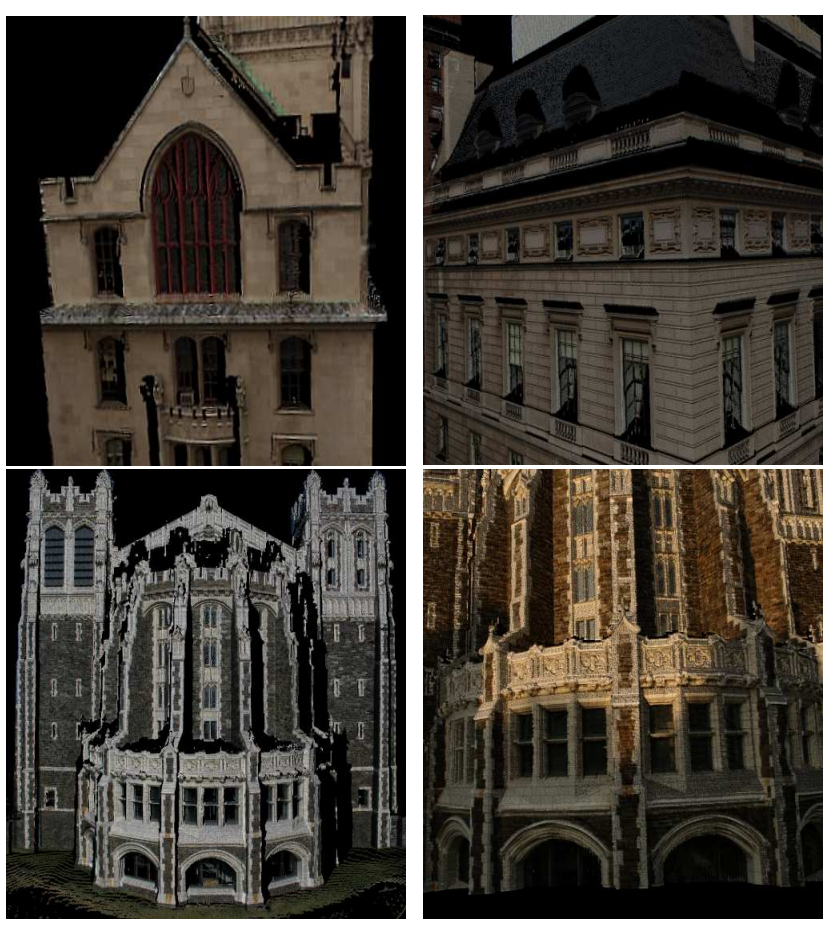

Figure 5. Details of texture-maps for buildings 1 (top left image), 2 (top right) and 3 (bottom two images) verifies the high accuracy of the automated algorithm. Note, that for building 3 we show results using images taken under different lighting conditions. 
(few pixels of translational adjustment) by a human user through our easy to use user interface.

The performance of our $3 \mathrm{D}$ range to $2 \mathrm{D}$ image registration algorithm is shown in Table 1 . The following abbreviations are being used. FP: Number of feature pairs (number

Table 1. Building 1 (11 images, 12 3D scans) - Building 2 (6 images, 3 range scans) - Building 3 (6 images, 4 range scans)

\begin{tabular}{cccccc}
\hline \hline FP(3D $\times 2 D)$ & CM & G & OP(\%) & RDT(\%) & T \\
\hline $117 \times 100$ & 25 & 14 & 91.23 & 0.21 & $42 \mathrm{sec}$ \\
$34 \times 54$ & 9 & 13 & 95.67 & 0.49 & $2 \mathrm{sec}$ \\
$117 \times 55$ & 28 & 17 & 93.45 & 0.43 & $11 \mathrm{sec}$ \\
$117 \times 145$ & 8 & 20 & 92.34 & 0.04 & $85 \mathrm{sec}$ \\
$117 \times 106$ & 15 & 9 & 91.23 & 0.12 & $44 \mathrm{sec}$ \\
$117 \times 44$ & 19 & 11 & 92.05 & 0.37 & $4 \mathrm{sec}$ \\
$117 \times 74$ & 7 & 32 & 94.56 & 0.08 & $21 \mathrm{sec}$ \\
$117 \times 113$ & 20 & 18 & 87.14 & 0.15 & $55 \mathrm{sec}$ \\
$35 \times 24$ & 1 & 16 & 98.40 & 0.1 & $0.6 \mathrm{sec}$ \\
$117 \times 74$ & 14 & 16 & 93.78 & 0.17 & $18 \mathrm{sec}$ \\
$35 \times 59$ & 9 & 5 & 89.31 & 0.43 & $1.5 \mathrm{sec}$ \\
\hline \hline $52 \times 80$ & 4 & 13 & 92.79 & 0.09 & $4 \mathrm{sec}$ \\
$63 \times 55$ & 155 & 18 & 96.13 & 4.4 & $35 \mathrm{sec}$ \\
$63 \times 46$ & 153 & 22 & 90.17 & 5.2 & $38 \mathrm{sec}$ \\
$52 \times 81$ & 8 & 8 & 86.38 & 0.19 & $5 \mathrm{sec}$ \\
$52 \times 61$ & 4 & 9 & 93.14 & 0.12 & $3 \mathrm{sec}$ \\
$52 \times 57$ & 18 & 7 & 92.23 & 0.61 & $4 \mathrm{sec}$ \\
\hline \hline $67 \times 60$ & 12 & 8 & 87.62 & 0.29 & $6 \mathrm{sec}$ \\
$67 \times 81$ & 18 & 8 & 92.45 & 0.31 & $10 \mathrm{sec}$ \\
$67 \times 39$ & 18 & 11 & 94.34 & 0.69 & $3 \mathrm{sec}$ \\
$67 \times 38$ & 12 & 11 & 91.74 & 0.47 & $2 \mathrm{sec}$ \\
$67 \times 71$ & 23 & 7 & 88.16 & 0.48 & $9 \mathrm{sec}$ \\
$67 \times 33$ & 7 & 8 & 93.51 & 0.31 & $1 \mathrm{sec}$ \\
\hline
\end{tabular}

of 3D features $\times$ number 2D features), CM: Number of candidate translations in list $\mathcal{T}$ after thresholding (see sixth step of algorithm of Sec. 4), G: Grade of optimum translation $\mathbf{t}_{\text {opt }}$ (number of matching features), OP: Amount of overlap among matching features (see fifth step of algorithm of Sec. 4), RDT: Percentage of candidate translations (CM) over all possible translations generated by matches of 3D and 2D features, T: Execution time of the automated registration algorithm on a $2 \mathrm{GHz}$ Pentium machine. The fast execution time is based on the large reduction of candidate translations (see RDT column).

In this paper, we presented a novel and efficient algorithm for the $3 \mathrm{D}$ range to $2 \mathrm{D}$ image registration problem in urban scene settings. Our input is a set of 3D range scans and a set of 2D images. The range scans are abstracted into sets of 3D lines, followed by three clustering steps. As a result, sets of $3 \mathrm{D}$ features are extracted. For each 2D image, features are generated via vanishing point extraction, camera calibration and rectification steps. Finally, an automated algorithm computes an optimized transformation between the $2 \mathrm{D}$ images and $3 \mathrm{D}$ range scans. This transformation is based on a match of 3D with $2 \mathrm{D}$ features that maximizes an overlap criterion. Our algorithm attacks the hard $3 \mathrm{D}$ range to $2 \mathrm{D}$ image registration problem in a systematic, efficient, and automatic way, in the context of urban scenes. Images captured by a high-resolution 2D camera, which moves and adjusts freely, are mapped on a centimeter-accurate 3D model of the scene providing photorealistic renderings of high quality.

\section{References}

[1] MIT City Scanning Project, 2004. http://city.lcs.mit.edu/.

[2] Visual Information Technology Group, Canada, 2004. http://iit-iti.nrc-cnrc.gc.ca/about-sujet/vit-tiv_e.html.

[3] T. Cass. Polynomial-time geometric matching for object recognition. IJCV, 21(1-2):37-61, 1997.

[4] C. Chen and I. Stamos. Semi-automatic range to range registration: a feature-based method, June 2005. To appear at 3DIM.

[5] S. Christy and R. Horaud. Iterative pose computation from line correspondences. CVIU, 73(1):137-144, January 1999.

[6] M. A. Fischler and R. C. Bolles. Random sample consensus: A paradigm for model fitting with applications to image analysis and automated cartography. Graphics and Image Processing, 24(6):381-395, June 1981.

[7] C. Früh and A. Zakhor. Constructing 3D city models by merging aerial and ground views. CGA, 23(6):52-11, 2003.

[8] R. Hartley and A. Zisserman. Multiple View Geometry in Computer Vision, second edition. Cambridge University Press, 2003.

[9] G. Hausler and D. Ritter. Feature-based object recognition and localization in 3D-space, using a single video image. CVIU, 73(1):64-81, 1999.

[10] R. Horaud, F. Dornaika, B. Lamiroy, and S. Christy. Object pose: The link between weak perspective, paraperspective, and full perspective. IJCV, 22(2), 1997.

[11] D. Huttenlocher and S. Ullman. Recognizing solid objects by alignment with an image. IJCV, 5(7):195-212, 1990.

[12] K. Ikeuchi. The great buddha project. In IEEE ISMAR03, Tokyo, Japan, November 2003.

[13] D. W. Jacobs. Matching 3-D models to 2-D images. IJCV, 21(1-2):123-153, 1997.

[14] F. Jurie. Solution of the simultaneous pose and correspondence problem using gaussian error model. CVIU, 73(3):357-373, March 1999.

[15] Leica Geosystems. http://hds.leica-geosystems.com/.

[16] D. G. Lowe. Distinctive image features from scale-invariant keypoint. IJCV, 60(2):91-110, 2004.

[17] D. Oberkampf, D. DeMenthon, and L. Davis. Iterative pose estimation using coplanar feature points. CVGIP, 63(3), May 1996. 
[18] C. Olson. Time and space efficient pose clustering. In CVPR, pages 251-258, Seattle, WA, 1994.

[19] K. Pulli, H. Abi-Rached, T. Duchamp, L. G. Shapiro, and W. Stuetzle. Acquisition and visualization of colored 3-D objects. In ICPR, Australia, 1998.

[20] L. Quan and Z. Lan. Linear N-point camera pose determination. PAMI, 21(7), July 1999.

[21] V. Sequeira and J. Concalves. 3D reality modeling: Photorealistic 3D models of real world scenes. In 3DPVT, pages 776-783, 2002.

[22] I. Stamos and P. K. Allen. Automatic registration of 3-D with 2-D imagery in urban environments. ICCV, pages 731736, 2001.

[23] I. Stamos and P. K. Allen. Geometry and texture recovery of scenes of large scale. Comput. Vis. Image Underst., 88(2):94-118, 2002.

[24] I. Stamos and M. Leordeanu. Automated feature-based range registration of urban scenes of large scale. CVPR, 2:555-561, 2003.

[25] W. Wells. Statistical approaches to feature-based object recognition. IJCV, 21(1-2):63-98, 1997.

[26] H. Zhao and R. Shibasaki. Reconstructing a textured CAD model of an urban environment using vehicle-borne laser range scanners and line cameras. MVA, 14(1):35-41, 2003.

[27] W. Zhao, D. Nister, and S. Hsu. Alignment of continuous video onto 3D point clouds. CVPR, pages 964-971, 2004. 